\title{
NONCLASSICAL GODEAUX SURFACES IN CHARACTERISTIC FIVE
}

\author{
RICK MIRANDA ${ }^{1}$
}

\begin{abstract}
A classical Godeaux surface is a smooth minimal projective surface $X$, with $K_{X}^{2}=1, p_{a}=p_{g}=0$ and $\operatorname{Pic}^{\tau}(X)=\mathrm{Z} / 5 \mathrm{Z}$. A nonclassical Godeaux surface is a smooth minimal projective surface $X$ with $K_{X}^{2}=1$, $p_{a}=0, p_{g}=1$ and $\operatorname{Pic}^{\tau}(X)=\mu_{5}$ or $\alpha_{5}$; such surfaces should exist in characteristic 5. It is the purpose of this note to construct nonclassical Godeaux surfaces in characteristic 5 , with $\operatorname{Pic}^{\tau}(X)=\mu_{5}$. The method is to exhibit a smooth quintic surface on which $\mathbf{Z} / 5 \mathbf{Z}$ acts, so that the quotient is smooth; this quotient is the desired surface.
\end{abstract}

Let $k$ be an algebraically closed field of characteristic 5 . Choose coordinates $[x, y, z, w]$ for $\mathbf{P}_{k}^{3}$; this allows us to identify $\operatorname{Aut}_{k}\left(\mathbf{P}^{3}\right)$ with $\operatorname{PGL}(3, k)$, which I will do. Let $\sigma$ be the automorphism represented by the matrix

$$
\left(\begin{array}{llll}
1 & 1 & 0 & 0 \\
0 & 1 & 1 & 0 \\
0 & 0 & 1 & 1 \\
0 & 0 & 0 & 1
\end{array}\right)
$$

This element has order 5 and generates a cyclic group $\langle\sigma\rangle$ isomorphic to $\mathbf{Z} / 5 \mathbf{Z}$ on $\mathbf{P}^{3}$. It is not difficult to see that every action of $\mathbf{Z} / 5 \mathbf{Z}$ on $\mathbf{P}^{3}$ which has only isolated fixed points is conjugate to the action of $\langle\sigma\rangle$. The only fixed point of $\sigma$ is $[1,0,0,0]$.

For any linear form $l$ in $x, y, z$ and $w$ let $N(l)=\prod_{i=0}^{4} \sigma^{i}(l)$ denote the norm of $l$ under the action of $\sigma ; N(l)$ is a quintic form which is invariant under $\sigma$. Let $V$ be the subspace of the space of quintic forms which is generated by norms of linear functions. Let $\phi: \mathbf{P}^{3} \rightarrow \mathbf{P}(V)$ be the (a priori rational) map defined by the subspace $V$. I claim that, in fact, $\phi$ is defined everywhere and is étale away from the fixed point $[1,0,0,0]$. The argument proceeds in several steps.

Let $C=\{\alpha \in \operatorname{PGL}(3) \mid \alpha \sigma=\sigma \alpha\}$ be the centralizer of $\sigma$. Then

$$
C=\left\{\left(\begin{array}{cccc}
a & b & c & d \\
0 & a & b & c \\
0 & 0 & a & b \\
0 & 0 & 0 & a
\end{array}\right) \mid a \neq 0\right\}
$$

and the action of $C$ on $\mathbf{P}^{3}$ decomposes $\mathbf{P}^{3}$ into 4 orbits $A_{0}, A_{1}, A_{2}$ and $A_{3}$, where $A_{0}=\{[1,0,0,0]\}$ is the fixed point, $A_{1}=\{[x, 1,0,0]\}, A_{2}=\{[x, y, 1,0]\}$ and

Received by the editors May 23, 1983 and, in revised form, August 25, 1983.

1980 Mathematics Subject Classification. Primary 14J25.

${ }^{1}$ Partially supported by NSF Grant No. MCS-82-02021. 
$A_{3}=\{[x, y, z, 1]\}$. Note that $C$ acts on forms in $x, y, z$ and $w$ in the standard way.

LEMMA 1. (1.1) If $f$ is a quintic form in $x, y, z$ and $w$ which is invariant under $\sigma$, and $\alpha \in C$, then $\alpha \cdot f$ is an invariant quintic.

(1.2) Let $p \in \mathbf{P}^{3}$, and assume that there exists an invariant quintic form $f$ such that $f(p) \neq 0$. Then for any $q$ in the orbit of $p$ under $C$, there exists an invariant quintic form $g$ such that $g(q) \neq 0$.

(1.3) Let $\left(p_{1}, p_{2}\right) \in \mathbf{P}^{3} \times \mathbf{P}^{3}$ and assume that there exists an invariant quintic form $f$ such that $f\left(p_{1}\right)=0$ and $f\left(p_{2}\right) \neq 0$. Then for any $\left(q_{1}, q_{2}\right)$ in the orbit of $\left(p_{1}, p_{2}\right)$ under $C$ (using the diagonal action), there exists an invariant quintic form $g$ such that $g\left(q_{1}\right)=0$ and $g\left(q_{2}\right) \neq 0$.

(1.4) Let $p \in \mathbf{P}^{3}$ and assume that the space $V$ of norms of linear functions separates tangent vectors at $p$. Then $V$ separates tangent vectors at any $q$ in the same orbit of $p$ under $C$.

PROOF. The first statement is trivial; the rest follow from it, using the general principle that if an invariant $f$ has a certain property at $p$, and if $\alpha \in C$, then the invariant $\alpha^{-1} \cdot f$ has that property at $q=\alpha \cdot p$. Q.E.D.

LEMMA 2. V has no base locus.

Proof. By (1.2), we need only check that one point in each orbit of $C$ is not a base point for $V$. It is easily seen that $N(x)$ is not zero at $[1,0,0,0] \in A_{0}, N(y)$ is not zero at $[0,1,0,0] \in A_{1}, N(z)$ is not zero at $[0,0,1,0]$, and $N(w)=w^{5}$ is not zero at $[0,0,0,1]$. Q.E.D.

LEMMA 3. $V$ separates the orbits of $\langle\sigma\rangle$.

PROOF. Assume that $p$ and $q$ are in different orbits of $\langle\sigma\rangle$. By (1.3), we may assume that $p=[1,0,0,0],[0,1,0,0],[0,0,1,0]$ or $[0,0,0,1]$, and (by symmetry) that the dimension of the $C$-orbit of $q$ is no greater than that of the $C$-orbit of $p$. This eliminates $p=[1,0,0,0]$ from consideration and leaves three cases.

Case 1. $p=[0,0,0,1], q=\left[x_{0}, y_{0}, z_{0} \cdot w_{0}\right]$. In this case write $\sigma^{i} \cdot q=\left[x_{i}, y_{i}, z_{i}, w_{i}\right]$ for $0 \leq i \leq 4$. Since $q$ is not in the orbit of $p$ under $\langle\sigma\rangle,\left(x_{i}, y_{i}, z_{i}\right) \neq(0,0,0)$ for any $i$. Hence there is a linear form $l(x, y, z)$ such that $l\left(x_{i}, y_{i}, z_{i}\right) \neq 0$ for $0 \leq i \leq 4$. Then $f=N(l)$ is in $V, f(q) \neq 0$ and $f(p)=0$.

Case 2. $p=[0,0,1,0], q=\left[x_{0}, y_{0}, z_{0}, 0\right]$. Using the same technique as in Case 1 , and writing $\sigma^{i} \cdot q=\left[x_{i}, y_{i}, z_{i}, 0\right]$, one can choose a linear form $l(x, y)$ such that if $f=N(l)$, then $f(q) \neq 0$ and $f(p)=0$.

Case 3. $p=[0,1,0,0], q=\left[x_{0}, y_{0}, 0,0\right]$. In this case $f=N(x)$ vanishes at $p$ and $f(q) \neq 0$. Q.E.D.

LEMMA 4. $V$ separates tangent vectors at all $p \neq[1,0,0,0]$ in $\mathbf{P}^{3}$.

Proof. By (1.4), we need only check this at one point in each orbit $A_{1}, A_{2}$ and $A_{3}$ of $C$. Again there are 3 cases to consider.

Case 1. $p=[0,0,0,1]$. In this case a computation shows that $N(a x+b y+c z)$ has, at $p$, the linear term

$$
(a x+b y+c z)[c(b+2 c)(a+3 b+3 c)(4 a+b+4 c)]
$$

which is general, for general $a, b$ and $c$. 
Case 2. $p=[0,0,1,0]$. Here the linear term of $N(a x+b y+c w)$ at $p$ is

$$
(a x+b y+c w)[b(a+2 b)(3 a+3 b)(a+4 b)]
$$

which is general, for general $a, b$ and $c$.

Case 3. $p=[0,1,0,0]$. Here the linear term of $N(a x+b z+c w)$ at $p$ is $(a x+b z+c w)\left[4 a^{4}\right]$ and is therefore general. Q.E.D.

The previous lemmas provide exactly what is needed to verify the claim and the proof of the following is complete.

Proposition 5. The map $\phi: \mathbf{P}^{3} \rightarrow \mathbf{P}(V)$ is a regular map which is étale away from the fixed point $[1,0,0,0]$ and separates the orbits of $\langle\sigma\rangle$.

Corollary 6. The image $Z$ of $\mathbf{P}^{3}$ under the map $\phi$ is the quotient of $\mathbf{P}^{3}$ under the action of $\langle\sigma\rangle$, and is smooth except possibly at $\phi([1,0,0,0])$.

COROLlARY 7. There exists a smooth quintic surface $Y$ in $\mathbf{P}^{3}$ which is invariant under $\langle\sigma\rangle$.

PROOF. By Bertini's theorem, there exists a smooth hyperplane section $X$ of $Z$ in $\mathbf{P}(V)$ since $Z$ has only one isolated singularity. The quintic surface is the pull-back of $X$ to $\mathbf{P}^{3}$. Q.E.D.

Of course, the surface $X$ is the quotient of $Y$ by $\langle\sigma\rangle$ and is the alleged nonclassical Godeaux surface. It remains to compute the invariants of the general such $X$. Let $\pi: Y \rightarrow X$ be the quotient map. By $[\mathbf{1}$, Theorem 2.1],

$$
\hat{\mathbf{Z}}_{5}=\mu_{5} \cong \operatorname{ker}(\pi: \operatorname{Pic} X \rightarrow \operatorname{Pic} Y),
$$

where $\hat{G}$ denotes the Cartier dual of a finite group scheme $G$. This is precisely $\operatorname{Pic}^{\tau}(X)$, since $Y$ is simply connected and $H^{0}\left(Y, \Omega_{Y}^{1}\right)=0$ (see [1, Example 2.3]). Therefore, $H^{1}\left(X, O_{X}\right)$ has dimension one. Since $\pi$ is étale, $c_{2}(X)=\frac{1}{5} c_{2}(Y)=11$, and $K_{Y}=\pi^{*} K_{X}$, so that $K_{X}^{2}=\frac{1}{5} K_{Y}^{2}=1$. By Noether's formula $\chi\left(O_{X}\right)=1$, so $p_{g}(X)=1$ and $p_{a}(X)=0$.

Classical Godeaux surfaces have been constructed in all characteristics by W. Lang [2]. I wish to thank him for suggesting this problem. I am also indebted to M. Artin for a helpful conversation.

\section{REFERENCES}

1. S. T. Jensen, Picard groups of quotients by finite commutative group schemes, Math. Scand. 42 (1978), 197-210.

2. W. Lang, Classical Godeaux surfaces in characteristic p, Math. Ann. 256 (1981), 419-427.

Department of Mathematics, Colorado State University, Fort Collins, COLORADO 80523

Current address: Department of Mathematics, Tufts University, Medford, Massachusetts 02155 